\title{
An effect of side chain length on the solution structure of poly(9,9-dialkylfluorene)s in toluene
}

\author{
M. Knaapila ${ }^{\mathrm{a}, *}$, L. Almásy ${ }^{\mathrm{b}}$, V.M. Garamus ${ }^{\mathrm{c}}$, M.L. Ramos ${ }^{\mathrm{d}}$, L.L.G. Justino ${ }^{\mathrm{d}}$, F. Galbrecht ${ }^{\mathrm{e}}$, \\ E. Preis ${ }^{\mathrm{e}}$, U. Scherf ${ }^{\mathrm{e}}$, H.D. Burrows ${ }^{\mathrm{d}}$, A.P. Monkman ${ }^{\mathrm{f}}$ \\ ${ }^{a}$ MAX-lab, Lund University, POB 118, SE-22100 Lund, Sweden \\ ${ }^{\mathrm{b}}$ Research Institute for Solid State Physics and Optics, POB 49, Budapest-1525, Hungary

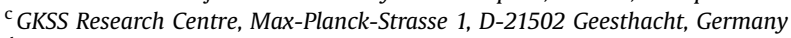 \\ ${ }^{\mathrm{d}}$ Departamento de Química, Universidade de Coimbra, P-3004-535 Coimbra, Portugal \\ e Fachbereich Chemie, Bergische Universität Wuppertal, Gauß-Strasse 20, D-42097 Wuppertal, Germany \\ ${ }^{\mathrm{f}}$ Department of Physics, University of Durham, South Road, DH1 3LE Durham, UK
}

\section{A R T I C L E I N F O}

Article history:

Received 9 November 2007

Received in revised form 22 February 2008

Accepted 27 February 2008

Available online 2 March 2008

\section{Keywords:}

Conjugated polymers

Polyfluorene

Solutions

\begin{abstract}
A B S T R A C T
The effect of side chain length of $\pi$-conjugated poly(9,9-dialkylfluorene)s has been studied in semi-dilute $(10 \mathrm{mg} / \mathrm{mL})$ toluene solutions using small-angle neutron scattering (SANS) and ${ }^{1} \mathrm{H}$ and ${ }^{2} \mathrm{H}$ NMR spectroscopies. Under these conditions, SANS data indicate that poly(9,9-dinonylfluorene) and poly(9,9dioctylfluorene) are dissolved down to the molecule level and appear as elongated one-dimensional chains (length $>20-30 \mathrm{~nm}$ ). In contrast, the shorter side chain polymers exhibit a self-association so that poly(9,9-diheptylfluorene) forms thin sheet-like $(\sim 1 \mathrm{~nm})$ and poly(9,9-dihexylfluorene) thin $(\sim 1 \mathrm{~nm})$ and thick sheet-like $(>6 \mathrm{~nm})$ aggregates. ${ }^{1} \mathrm{H}$ NMR data, together with the density functional theory (DFT) calculations, however, show that this occurs without changes in the conformation of the polymer backbone.
\end{abstract}

(c) 2008 Elsevier Ltd. All rights reserved.

\section{Introduction}

Polyfluorenes (PFs) [1-4] are a central class of $\pi$-conjugated polymers demonstrating myriad applications in solution processed systems for polymer electronics [5]. Their intermolecular, self-organizational behavior stems largely from the hairy rod type molecular structure where flexible side chains are covalently bonded to the relatively stiff backbone [6,7]. The resulting phase behavior has been extensively studied in the solid state but the literature of their solution assemblies remains relatively sparse, with a few specific exceptions such as poly[9,9-bis(2-ethylhexyl)-fluorene-2,7-diyl] (PF2/6) [8-10], corresponding oligomers [11], and poly(9,9-dialkylfluorene-co-fluorenone) copolymers [12] in toluene as well as poly\{2,7-[9,9-bis((S )-3,7-dimethyloctyl)]fluorene $\}$ in tetrahydrofuran [13]. Systematic studies on the various aspects of solution assemblies would be advantageous, not least because the solvent plays a major role in the morphology of solvent processed thin films [14].

The solution behavior of PFs can be rationalized in terms of three phenomenological variables - the nature of solvent, the volume

\footnotetext{
* Corresponding author. Tel.: +46 4622 24306; fax: +46 462224710 .

E-mail address: matti.knaapila@maxlab.lu.se (M. Knaapila).
}

fraction of polymer, and the nature of side chain. A further parameter which becomes dominant in the oligomeric limit is the length of the polymer [11]. Poly(9,9-dioctylfluorene) (PF8) [15] is perhaps the most thoroughly studied polyfluorene. In the solid state, PF8 is polymorphic with a range of crystalline [16] and non-crystalline [17] phases. Its chain morphology has been detailed in different organic solvents such as chloroform, toluene, tetrahydrofuran and cyclohexane [18] and dramatic structural diversity arises from the quality of solvent, the first parameter in our discussion. For example, PF8 forms sheet-like particles in $1 \%(\sim 10 \mathrm{mg} / \mathrm{mL})$ solution in the poor solvent methylcyclohexane $(\mathrm{MCH})$ but rodlike structures in the better solvent, toluene [10]. In addition, if the second parameter, the polymer fraction is increased from $1 \%$ to $3-7 \%(\mathrm{w} / \mathrm{v})$ in toluene, individual PF8 molecules aggregate and form a large network-like structure [19]. In our previous work [20] we have studied the effect of side chain length, the third variable, using poly(9,9-dihexylfluorene) (PF6), poly(9,9-diheptylfluorene) (PF7), PF8, poly(9,9-dinonylfluorene) (PF9), and poly(9,9-didecylfluorene) (PF10) in $\mathrm{MCH}$. In $\mathrm{MCH}$, all these polymers except PF10 form sheet-like structures at the colloidal level $(1-10 \mathrm{~nm})$ at $20^{\circ} \mathrm{C}$ at concentrations $1-5 \%(w / v)$ [20].

In this paper we report the effect of side chain length on the solution assemblies of PF6, PF7, PF8, and PF9 in toluene. Since it is a better solvent, toluene provides a standard point of comparison 
for $\mathrm{MCH}$. We find that improving the solvent quality leads to significant differences in nanoscale structure formation. PF8 and PF9 always dissolve down to the molecular level, adopting an elongated (rodlike) structure of a single polymer chain. In contrast, PF6 and PF7, with their shorter side chains, exist as sheet-like aggregates, this tendency being most pronounced for PF6. However, we will see that ${ }^{1} \mathrm{H}$ NMR spectroscopy, coupled with DFT calculations, shows that these structures are formed without any significant changes in the torsional angles between the fluorene rings of the backbone. While the way that aggregate formation of PFs is controlled by varying the concentration in toluene is known [19], we will show how this behavior can also be controlled by varying the side chain length. This information is expected to have implications when optimizing morphology in polyfluorene thin film devices prepared from this class of solvents.

\section{Experimental section}

PF6, with the number average molecular weight $\left(M_{\mathrm{n}}\right)=84 \mathrm{~kg} /$ mol, weight average molecular weight $\left(M_{\mathrm{W}}\right)=200 \mathrm{~kg} / \mathrm{mol}$, together with PF7 $\left(M_{\mathrm{n}}=63 \mathrm{~kg} / \mathrm{mol}, M_{\mathrm{W}}=144 \mathrm{~kg} / \mathrm{mol}\right), \operatorname{PF} 8\left(M_{\mathrm{n}}=48 \mathrm{~kg} / \mathrm{mol}\right.$, $\left.M_{\mathrm{w}}=132 \mathrm{~kg} / \mathrm{mol}\right)$, and PF9 $\left(M_{\mathrm{n}}=109 \mathrm{~kg} / \mathrm{mol}, M_{\mathrm{w}}=221 \mathrm{~kg} / \mathrm{mol}\right)$ (Fig. 1) were prepared following the Yamamoto-type polymerization with $\mathrm{Ni}(\mathrm{COD})_{2}$ catalyst [2]. The polymers were mixed in $10 \mathrm{mg} / \mathrm{mL}$ (or in 2 cases $3 \mathrm{mg} / \mathrm{mL}$ ) deuterated toluene (toluene$d_{8}$ ) $(99.5 \% \mathrm{D}$, Cambridge Isotope Laboratories Inc.). All polymers except PF6 were dissolved by stirring for $5 \mathrm{~min}$ at $20^{\circ} \mathrm{C}$. PF6 is not completely soluble in toluene at room temperature but can be dissolved by heating quickly up to $84{ }^{\circ} \mathrm{C}$ and cooling slowly down to $20^{\circ} \mathrm{C}$. Mixtures in toluene- $d_{8}$ were then measured as such.

Small-angle neutron scattering (SANS) measurements were performed using both the Yellow Submarine instrument at the BNC in Budapest (Hungary) and the SANS-1 instrument at the GKSS Research Centre in Geesthacht (Germany). The overall $q$-range was from 0.004 to $0.4 \AA^{-1}$. The samples were filled in Hellma quartz cells of $2 \mathrm{~mm}$ path length and placed in a thermostatic holder. The raw scattering patterns were corrected for sample transmission, room background, and sample cell scattering. The isotropic 2-dimensional scattering patterns were azimuthally averaged, converted to an absolute scale and corrected for detector efficiency dividing by the incoherent scattering spectra of $1 \mathrm{~mm}$ thick pure water. The scattering from toluene- $d_{8}$ used for the sample preparation was subtracted as a background; the small incoherent scattering due to the non-deuterated polymer was taken into account by fitting procedure.

${ }^{1} \mathrm{H}$ and ${ }^{2} \mathrm{H}$ NMR spectra of PFs in toluene- $d_{8}$ were obtained at $25{ }^{\circ} \mathrm{C}$ on a Varian Unity-500 NMR spectrometer at $499.824 \mathrm{MHz}$ $\left({ }^{1} \mathrm{H}\right)$ and $76.726 \mathrm{MHz}\left({ }^{2} \mathrm{H}\right)$, using spectral widths of $5000 \mathrm{~Hz}\left({ }^{1} \mathrm{H}\right)$ and $766.6 \mathrm{~Hz}\left({ }^{2} \mathrm{H}\right)$, acquisition times of $4 \mathrm{~s}$, pulse delays of $3 \mathrm{~s}\left({ }^{1} \mathrm{H}\right)$ and $0.1 \mathrm{~s}\left({ }^{2} \mathrm{H}\right)$ and about 320 pulses, at 25,45 , and $65^{\circ} \mathrm{C}$. The methyl residual solvent signal centred at $2.09 \mathrm{ppm}$ was used as internal reference; all chemical shifts are given with respect to tetramethylsilane (TMS).

\section{Computational section}

For analysis of the effect of inter-ring torsion on the chemical shifts in the NMR spectra of PFs, the molecular structure of the model

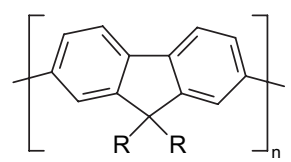

Fig. 1. The chemical structure of PFs studied; $\mathrm{R}=\left(\mathrm{CH}_{2}\right)_{x} \mathrm{CH}_{3} ; x=5,6,7$, and 8 for PF6, PF7, PF8, and PF9, respectively. compound biphenyl was optimized by density functional theory (DFT) prior to calculation of the nuclear shielding. The optimization was performed without symmetry constraints using GAMESS [21]. The B3LYP functional (Becke three-parameter Lee-Yang-Parr exchange correlation functional), which combines the hybrid exchange functional of Becke [22] with the correlation functional of Lee, Yang and Parr [23] was used. The 6-31G(d,p) basis sets [24] were used for the expansion of the Kohn-Sham orbitals. At the equilibrium geometry the maximum gradient was $10^{-5}$ Hartree bohr ${ }^{-1}$. Using the optimized geometry, the $\mathrm{C} 2 \mathrm{C} 1 \mathrm{C}^{\prime} \mathrm{C} 2^{\prime}$ torsion angle has been varied at $10^{\circ}$ intervals and the nuclear shieldings have been computed for these different geometries. These were computed with the uncoupled DFT (UDFT) approach $[25,26]$ using individual gauges for localized orbitals (IGLO). The calculations were carried out with the deMon program [27] (including the deMon-NMR modules). The calculations employed the PW91 functional $[28,29]$ and the IGLO-II basis sets. ${ }^{1} \mathrm{H}$ chemical shifts are given with respect to the absolute shielding value of TMS obtained at the same computational level.

\section{Results and discussion}

In Fig. 2 we plot the SANS data of PF6, PF7, PF8, and PF9 in $10 \mathrm{mg} /$ $\mathrm{mL}$ toluene- $d_{8}$ measured at $20^{\circ} \mathrm{C}$. In general, all data can be described in terms of one or two characteristic slopes over the $q$-range up to $q=0.2 \AA^{-1}$. Above this point, the scattering patterns decay more steeply and reach the level of the incoherent background. This value seems to change for the different samples, which is attributed to slight differences in the concentrations used, enhanced by subtraction of the same background of pure toluene- $d_{8}$. The higher $q$ part for all four samples shows the characteristic pattern of elongated cylindrical objects. Differences between the samples are observed at the lower $q$-range. The data of PF8 and PF9 in toluene- $d_{8}$ are essentially the same and are dominated by the -1 decay over the whole $q$-range, while data for PF6 and PF7 show an abrupt upturn at about $q \sim 0.06 \AA^{-1}$, with their lower $q$ part following another linear behavior with a slope of -2.3 . These data are conveniently explained by models of one-dimensional rodlike objects and two-dimensional sheet-like objects, which give, respectively, the slopes of -1 and -2 in the log-log presentation. For the two shorter side chain polymers, the data thus indicate the coexistence of sheet-like aggregates and individual rodlike chains. Such

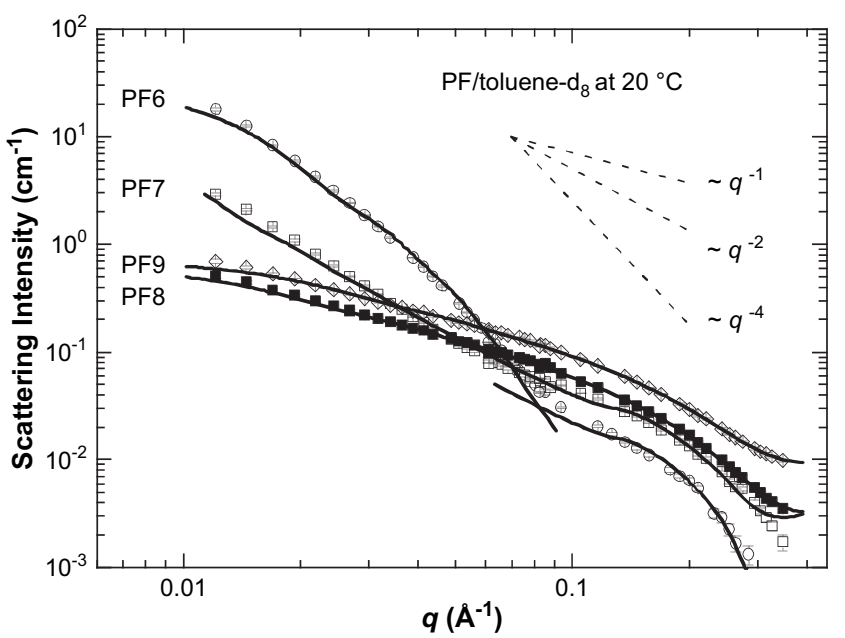

Fig. 2. SANS data of PF6 (open circles), PF7 (open squares), PF8 (solid squares), and PF9 (open diamonds) in toluene- $d_{8}$ at $20.0 \pm 0.5^{\circ} \mathrm{C}$. Solid lines plot the fits to the appropriate models of simple objects. Note that for PF6 separate models have been applied for the low and high $q$ parts. Dashed lines show the $-1,-2$, and -4 decays for comparison. The concentrations were $10 \mathrm{mg} / \mathrm{mL}$. 


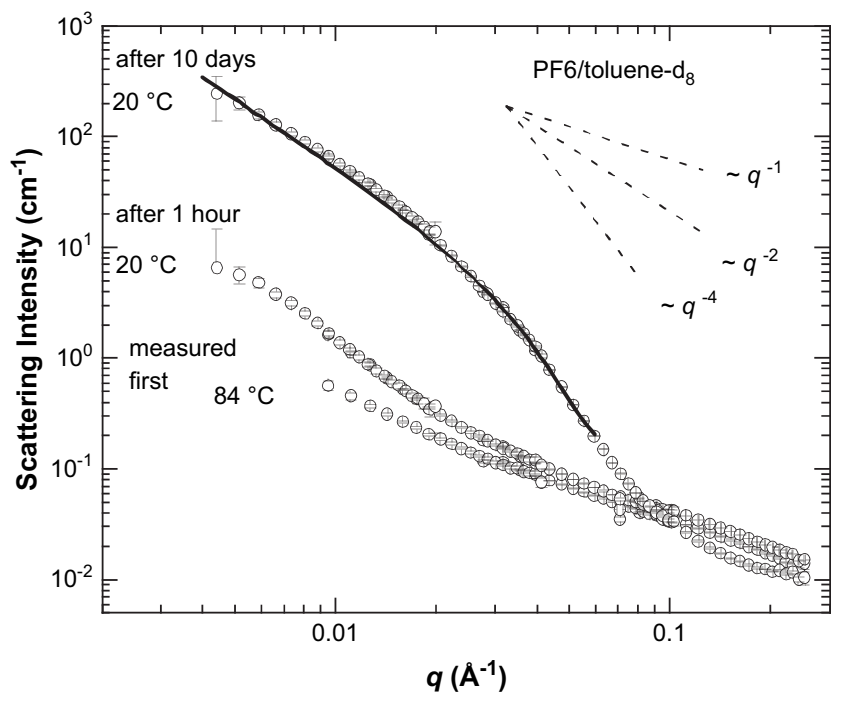

Fig. 3. SANS data of PF6 in toluene- $d_{8}$ at $84 \pm 1{ }^{\circ} \mathrm{C}$ as well as the data at $20.0 \pm 0.5^{\circ} \mathrm{C}$ when measured ca. $1 \mathrm{~h}$ and 10 days after the measurement at $84^{\circ} \mathrm{C}$. Dashed lines show the $-1,-2$, and -4 decays for comparison. A solid line plots the fit to the sheet-like model. The concentration was $10 \mathrm{mg} / \mathrm{mL}$.

coexistence is obviously sensitive to external parameters, and probably in addition to the preparation conditions and history. In order to probe this, PF6 and PF7 samples were measured at elevated temperatures.

Fig. 3 plots SANS data of PF6 in $10 \mathrm{mg} / \mathrm{mL}$ toluene- $d_{8}$ at $84{ }^{\circ} \mathrm{C}$ as well as the data of the same sample at $20^{\circ} \mathrm{C}$ as measured $1 \mathrm{~h}$ and 10 days after annealing. At the elevated temperature PF6/toluene- $d_{8}$ shows a fair slope of -1 over the whole observation window, with only a weak sign of -2 scaling being visible at $q<0.02 \AA^{-1}$. When the sample is allowed to cool slowly down to $20^{\circ} \mathrm{C}$ and measured for a second time after $1 \mathrm{~h}$, the PF6/toluene- $d_{8}$ mixture shows an aggregate formation as indicated by a distinct upturn and subsequent slope of -2 below $0.02 \AA^{-1}$. The aggregate formation continues and is better distinguished after 10 days when the data are fairly similar to the original ones (Fig. 2). In contrast, PF7/toluene$d_{8}$ solution shows slope of -1 at $84^{\circ} \mathrm{C}$ and this picture is not altered for 10 days after cooling (not shown).

The simple interpretation of the observed scattering functions using scaling concepts can be enhanced by numerical modeling to certain geometric shapes. The data have been fitted by models of cylinder-like and sheet-like objects [30]; the fits are shown in Fig. 2 and Fig. 3, and the relevant parameters are collected in Table 1. For PF6/toluene sample exhibiting coexistent phases, the fitting was performed on the appropriate restricted $q$-ranges.

The characteristic scattering patterns obtained allowed us to attribute them to various structural arrangements of the polymers in solutions. Fig. 4 illustrates three types of behavior of small-angle scattering data and proposed levels of solution structures of PF/

Table 1

Parameters fitted to the SANS data of PF/toluene- $d_{8}$ systems with concentration of $10 \mathrm{mg} / \mathrm{mL}$

\begin{tabular}{lllllll}
\hline $\begin{array}{l}\text { Polymer } \\
\text { in MCH }\end{array}$ & Model & $\begin{array}{l}\text { Analyzed } \\
q \text {-range }\left(\AA^{-1}\right)\end{array}$ & $T(\AA)$ & $R(\AA)$ & $D(\AA)$ & $L(\AA)$ \\
\hline PF6 & Sheet & $0.01-0.1$ & $56 \pm 4$ & n/a & $>1000$ & n/a \\
PF6 & Sheet & $0.1-0.4$ & $10 \pm 2$ & n/a & $>300$ & n/a \\
PF6 after 10 days & Sheet & $0.004-0.06$ & $90 \pm 2$ & n/a & $>1000$ & n/a \\
PF7 & Sheet & $0.01-0.4$ & $10 \pm 2$ & n/a & $>600$ & n/a \\
PF8 & Cylinder & $0.01-0.4$ & n/a & $9.5 \pm 0.5$ & n/a & $>220$ \\
PF9 & Cylinder & $0.01-0.4$ & n/a & $9.4 \pm 0.5$ & n/a & $>290$ \\
\hline
\end{tabular}

$T, R, D$, and $L$ are the estimation of the thickness of the sheet, radius of the cylinder (rod), diameter of the sheet, and the length of the cylinder, respectively, obtained from the models fitted to the SANS data.

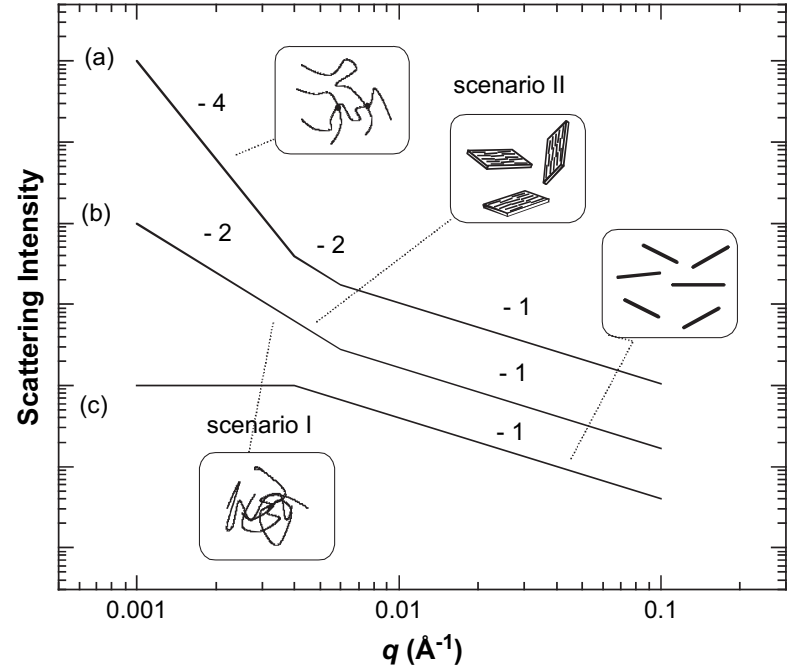

Fig. 4. Schematic description of the scattering data and proposed structures of PFs in toluene: (a) a network-like structure with cross-linked nodes; (b) a structure of rodlike particles forming Gaussian coils (scenario I) or sheet-like aggregates (scenario II) in the longer length scales, and (c) a structure of fully dissolved rodlike particles (essentially single polymer chains).

toluene mixtures. In all these schematic cases the apparent rigidity of PF backbone would lead to -1 scaling followed by steeper decay at high $q$, due to the finite polymer thickness (not shown). The differences between the outlined models are reflected at the lowest values of the experimental $q$-range. In all cases the polymers are considered as relatively stiff particles.

Case 1: If the mixture of rodlike chains included a network-like structure with cross-linked nodes, $q^{-4}$ scaling would be observed at low $q$. This model is illustrated in Fig. 4(a). The data corresponding to this situation are reported in Ref. [19] for PF8/toluene- $d_{8}$ solutions when the concentrations are $3 \%(\mathrm{w} / \mathrm{v})$ or higher. If the nodes were actually small crystallites, X-ray scattering data should show sharp reflections at wider angles, a model which has been put forward for PF7/MCH, PF8/MCH, and PF9/MCH mixtures [20]. The $q$ range employed in our study is unfortunately too narrow either to confirm or reject the Case 1 for the studied PF solutions.

Case 2: If the mixture consisted of interconnected rodlike particles forming either Gaussian chains (scenario I) or sheet-like particles (scenario II) at larger length scales, the data would correspond to that shown in Fig. 4(b). In this case polymer-polymer interactions begin to dominate and tangled chains cling together forming mazes over the larger intermolecular distances, although the mixture is not macrophase separated. Such experimental data are qualitatively seen for PF6 and PF7 in toluene- $d_{8}$ with the concentration of $10 \mathrm{mg} / \mathrm{mL}$ (Fig. 2). The parameters deducted from the data fitted to the model of sheet-like particles are compiled in Table 1. PF7/ toluene- $d_{8}$ forms thin sheets, presumably containing only one polymer layer. In contrast, it seems that the PF6/toluene- $d_{8}$ system may contain a polydisperse mixture of two kinds of sheets - thin ones with one polymer layer as well as thick ones composed of several polymer layers. The variation of sheet thicknesses is also present for polyfluorene sheets in $\mathrm{MCH}$ [20].

Case 3: If the mixture consisted of fully dissolved rodlike particles, then the data would correspond to the model shown in Fig. $4(\mathrm{c})$. In this case the -1 decay turns to a constant plateau at the lowest scattering angles. Such behavior is observed for PF8 and PF9 in toluene- $d_{8}$ with concentration of $10 \mathrm{mg} / \mathrm{mL}$ and good fits to the corresponding models of rodlike particles are obtained (solid lines in Fig. 2). The parameters deduced from the data fitted to the model of rodlike particles are depicted in Table 1 . The limited 
$q$-range does not allow the full inspection of Guinier regime and therefore the obtained lengths of the particles are estimations for the lowest limit. As the radius of particles is essentially that of single polymers $(\sim 1 \mathrm{~nm})$, these data are interpreted as arising from single rodlike chains dissolved down to the molecular level. Similar results have also been obtained for toluene solutions of PF2/6 [8-10], F2/6 oligomers [11], and fluorene-fluorenone random copolymers [12].

While the solution structures of PF8/toluene- $d_{8}$ and PF9/toluene- $d_{8}$ systems as fully dissolved stiff chains seem clear in the concentration and length scale studied, more interesting are the PF6/toluene- $d_{8}$ and PF7/toluene- $d_{8}$ mixtures in Case 2. Analogous scattering data with an upturn from -1 to -2 behavior have been reported for semi-stiff $\pi$-conjugated poly[2-methoxy-5(2'-ethyl-hexyloxy)-1,4-phenylene vinylene] in toluene [31] and poly(2,3-diphenyl-5-hexyl-1,4-phenylenevinylene) in chloroform [32]. In these reports the different asymptotic behavior in the various $q$-ranges is interpreted as the coexistence of linear wholly dissolved polymers ( -1 decay) and sheet-like aggregates ( -2 decay). We expect that this interpretation is also valid for the observed $q^{-2.3} \approx q^{-2}$ decay for $q<0.02 \AA^{-1}$ in PF6/toluene- $d_{8}$ and PF7/toluene- $d_{8}$, and believe that this scaling is an indication of larger sheet-like particles comparable to those found in $\mathrm{PF} / \mathrm{MCH}$ systems [20].

There are several reasons why the existence of sheet-like particles (scenario II) is more likely than that of Gaussian chains (scenario I). Firstly, the scattering of the Gaussian chains should be weaker, and not greater than that of rigid chains. Moreover, it is not plausible for stiff polymers to organize as Gaussian coils except at very long length scales. Regarding the length scale aspect, we point out that the -2 decay is observed for PF6/toluene- $d_{8}$ and PF7/toluene- $d_{8}$ mixtures for the interval $0.004 \AA^{-1}<q<0.06 \AA^{-1}$. In contrast, PF8/toluene- $d_{8}$ and PF9/toluene- $d_{8}$ obey -1 behavior down to the lowest limit of the observation window (Fig. 2). If the approximate -2 decay originated from Gaussian chains, it would be observed for all the PFs studied. We note in addition that even the shortest polymer is longer than $100 \mathrm{~nm}$ and thus the end-connected aggregates [11] are not expected to play a role. We also note that PF6/toluene- $d_{8}$ and PF7/toluene- $d_{8}$ mixtures exhibit a transition from -2 to -1 scattering characteristics around $70-80{ }^{\circ} \mathrm{C}$ and wish to argue that in this transition the sheets composed of stiff polymer chains are transformed to the phase consisting of completely dissolved chains in toluene- $d_{8}$. Any hypothetical "Gaussian chain - rodlike chain" transition with increasing temperature would involve an unphysical transformation from less ordered to more ordered phase, providing a further argument against the existence of Gaussian chains over sheet-like architecture.

Overall, though qualitative, these results show that the solution structure of polyfluorenes can be switched from rod to sheet simply by modifying the side chain length. The aggregation tendency is greatly reduced when changing solvent from $\mathrm{MCH}$ [20] to toluene. The distinctive differences in the optical data of various PF8 solutions (reported elsewhere [18]) suggest that the structural picture could be elaborated by varying the quality of solvent further still.

All small-angle scattering data probe inclusively long-range order at around $1-100 \mathrm{~nm}$. Therefore, in order to judge further whether scenario I or II is probable from the local $(<1 \mathrm{~nm})$ perspective, and to get an idea of potential differences in the local structure, we have performed a ${ }^{1} \mathrm{H}$ NMR study. We have already shown the complementarity of SANS and NMR in studying different length scales in solutions of conjugated polymers [33]. It is expected that these would show any differences in behavior of the four polymers in solution, and in addition may provide evidence for polymer-polymer interactions, which would be expected to be strong in the scenario II.

Fig. 5 plots ${ }^{1} \mathrm{H}$ NMR data of the mixtures studied. The spectrum of PF8 in perdeuterotoluene is similar to that previously reported in Ref. [19]. Assignment of the peaks in the four spectra is based on this work and the report by Leclerc and colleagues [34]. In all four systems, peaks due to residual solvent protons are observed around $2.1 \mathrm{ppm}$ and in the region 6.9-7.1 ppm. In addition, signals due to the alkyl chains were observed in the region $0.8-1.15 \mathrm{ppm}$, while the bands due to the fluorene aromatic protons were observed between 7.78 and $7.96 \mathrm{ppm}$. The aliphatic protons showed the expected changes in relative intensity on going from the hexyl (PF6) to nonyl (PF9) chains, but did not show any significant shifts. Similarly, the spectra corresponding to the fluorene aromatic protons were identical for the four compounds. This strongly suggests that these polymers have similar backbone conformations. To test the hypothesis further, we have carried out DFT calculations on the effect of torsion about the inter-ring $\mathrm{C}-\mathrm{C}$ bond on chemical shifts of these protons in the model compound biphenyl (Fig 6). The calculated equilibrium torsion angle in biphenyl $\left(38.07^{\circ}\right)$ is very close to that in fluorene dimer $\left(40.12^{\circ}\right)$.

As can be clearly seen from Fig. 6, if there were any significant differences in the torsion angles, marked changes in the chemical shifts of these protons would be expected. The fact that the spectra of PF6, PF7, PF8 and PF9 in toluene solution are effectively identical indicates that they have similar backbone conformations.

It has been suggested by Rahman et al. [19] that in the case of toluene- $d_{8}$ solutions of PF8, further insight on aggregate structures can be obtained by ${ }^{2} \mathrm{H}$ NMR. We have looked at the ${ }^{2} \mathrm{H}$ NMR spectra

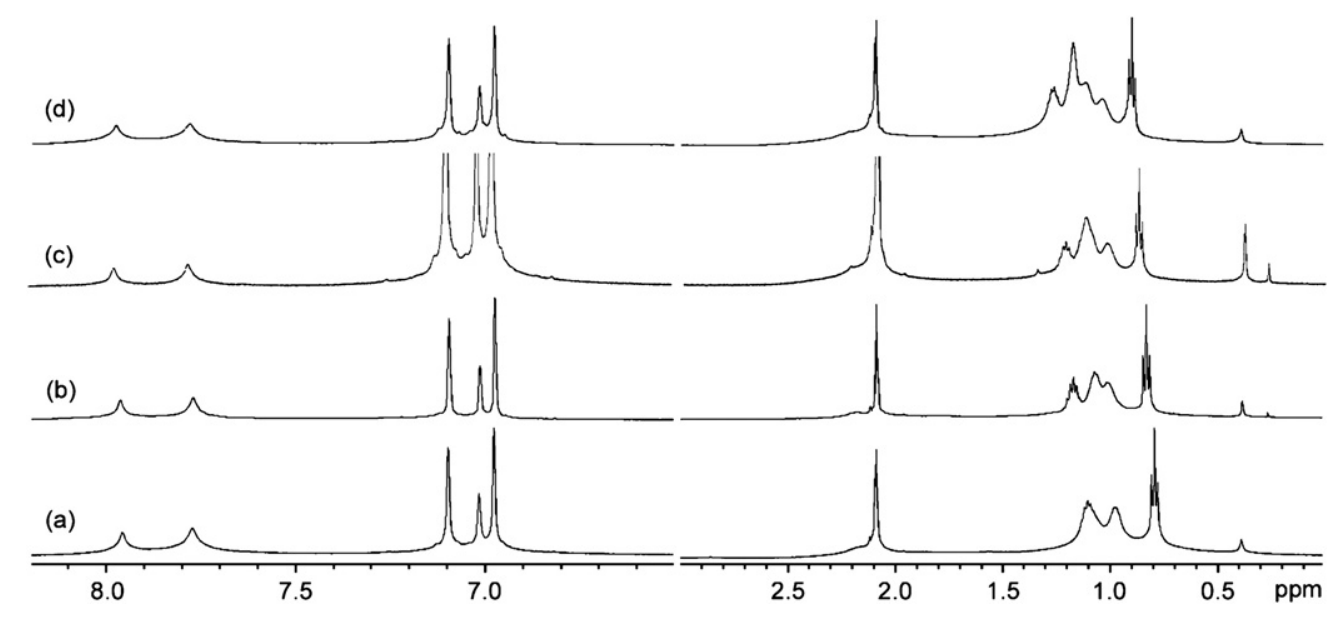

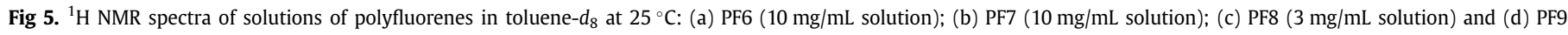
(10 $\mathrm{mg} / \mathrm{mL}$ solution). 


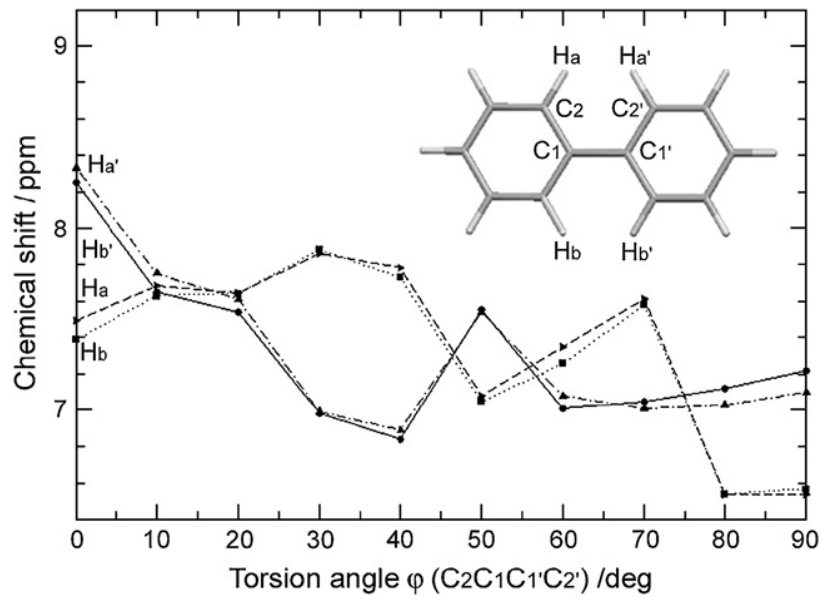

Fig 6. Torsion angle dependence of chemical shift for the $\mathrm{Ha}$ (dashed line), $\mathrm{Ha}^{\prime}$ (dashed dot line), $\mathrm{Hb}$ (dotted line) and $\mathrm{Hb}^{\prime}$ (solid line) hydrogen atoms of biphenyl calculated using the UDFT approach.

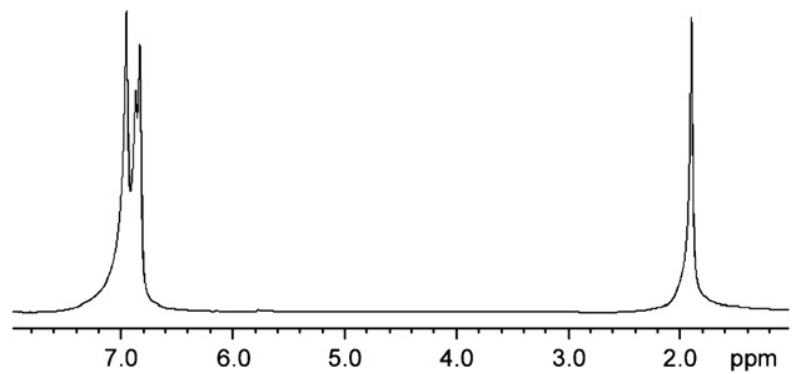

Fig. 7. ${ }^{2} \mathrm{H}$ NMR spectrum $(76.726 \mathrm{MHz})$ of a $30 \mathrm{mg} / \mathrm{mL}$ PF9 solution in toluene- $d_{8}$, at $25{ }^{\circ} \mathrm{C}$.

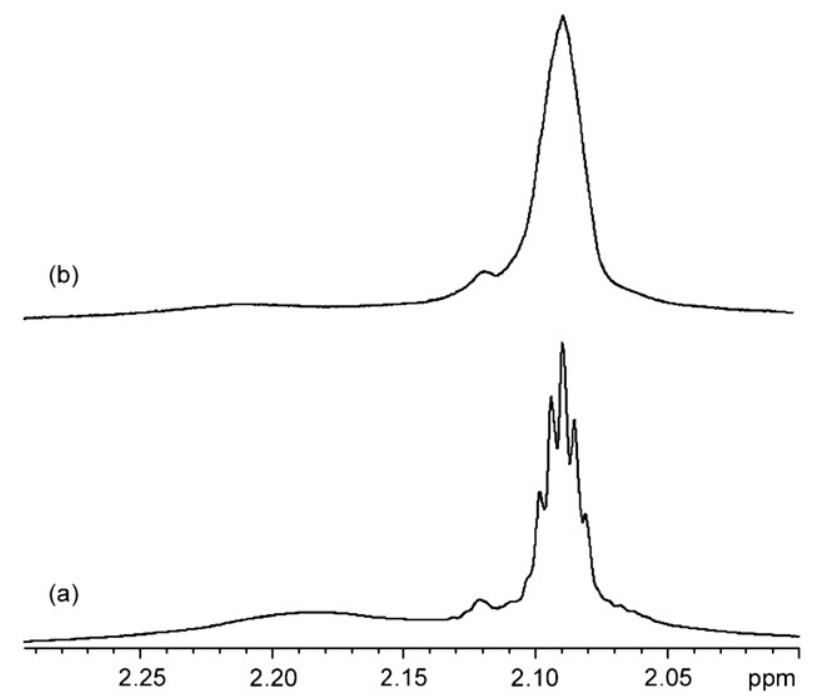

Fig. 8. Residual $\mathrm{CD}_{2} \mathrm{H}$ proton $\mathrm{NMR}$ resonances (499.824 MHz) of the solvent (toluene$d_{8}$ ) of (a) $10 \mathrm{mg} / \mathrm{mL}$ PF6 and (b) $3 \mathrm{mg} / \mathrm{mL}$ PF8 solutions, at $65{ }^{\circ} \mathrm{C}$.

of solutions of the four PFs in perdeuterotoluene and have not been able to detect any significant difference from that of the pure solvent under our conditions. The ${ }^{2} \mathrm{H}$ NMR spectrum for PF9 is shown in Fig 7. However, differences are observed in the signal observed for the residual methyl proton of the toluene- $d_{8}$ solvent in the ${ }^{1} \mathrm{H}$ NMR spectra of solutions of these polymers. This signal is coupled with deuterium and gives rise to a complex multiplet. Changes in the rotational motion of the solvent will be expected to affect the spectral bandwidth, and may lead to loss of this structure. Spectra were recorded in this region for the four polymer systems at temperatures between $25^{\circ}$ and $65^{\circ} \mathrm{C}$. Clear differences were observed in this region between the spectrum with PF8 and the polymers with shorter side chains. Results for two of the systems are presented in Fig. 8. Whilst detailed analysis of these spectral changes is outside the scope of this work, they are consistent with the view from the SANS measurements that the structure and dynamics of the aggregate solutions with PF6 is significantly different from that with PF8, and hence favour the scenario II, i.e., the sheet formation. We note that there can be effects with the backbone with a small fraction of the molecules but these are not necessarily seen as the spectra are dominated by the bulk signals.

\section{Conclusion}

Summarizing, the effect of side chain length on the solution assemblies of poly(9,9-dialkylfluorene)s has been investigated in semi-dilute toluene- $d_{8}$ solutions $(10 \mathrm{mg} / \mathrm{mL})$ using SANS and NMR. The solution structures show the gradual changes with decreasing side chain length of the polymers. In the larger intermolecular level ( $\sim 1-70 \mathrm{~nm}$ ) PF9 and PF8 are dissolved down to the molecular level and single (rodlike) polymer chains are observed. Instead, the shorter side chains of PF7 and PF6 lead in toluene to the emergence of sheet-like aggregates $(\sim 60 \mathrm{~nm})$. In the SANS data this deviation is manifested as a sudden upturn and subsequent -2 decay at $0.02 \AA^{-1}$. ${ }^{1} \mathrm{H}$ NMR spectra are very similar for all the polymer solutions, indicating no significant changes in the conformations of the polymer backbone. However, from the peak attributed to residual toluene- $d_{8}$ solvent methyl group in the ${ }^{1} \mathrm{H}$ NMR, there are indications of differences, which are consistent with fluorene-toluene contacts prevailing for PF8 whereas fluorene-fluorene contacts may be more important for PF7 and PF6 in $10 \mathrm{mg} / \mathrm{mL}$ toluene solution. As stiff chains cannot make loops on this length scale, this observation is in accord with the idea of sheet-like aggregates. Overall, though qualitative, these results interestingly show that the solution structure of a polyfluorene can be switched from rod to sheet simply by varying its side chain length.

\section{Acknowledgements}

We are indebted to M. Torkkeli of the University of Helsinki for fruitful discussions. M.K. thanks European Commission (Contract no. RII3-CT-2003-505925). L.A. acknowledges Marie Curie Network "POLYAMPHI". L.L.G.J. thanks FCT for the grant SFRH/BPD/26415/ 2006 and Centre for Computational Physics of Physics Department of University of Coimbra for the computing facilities. The Coimbra group is also grateful to $\mathrm{POCI} / \mathrm{FEDER} / \mathrm{FCT}$ for further financial support through the project POCI/QUI/58291/2004.

\section{References}

[1] Neher D. Macromol Rapid Commun 2001;22:1365.

[2] Scherf U, List EJW. Adv Mater 2002;14:477.

[3] Grimsdale AC, Müllen K. Adv Polym Sci 2006;199:1.

[4] Knaapila M, Stepanyan R, Lyons BP, Torkkeli M, Monkman AP. Adv Funct Mater 2006;16:599.

[5] Zaumseil J, Sirringhaus H. Chem Rev 2007;107:1296.

[6] Ballauff M. Angew Chem Int Ed 1989;28:253.

[7] Wegner G. Macromol Chem Phys 2003;204:347.

[8] Fytas G, Nothofer HG, Scherf U, Vlassopoulos D, Meier G. Macromolecules 2002;35:481.

[9] Knaapila M, Stepanyan R, Torkkeli M, Lyons BP, Ikonen TP, Almásy L, et al. Phys Rev E 2005;71:041802.

[10] Knaapila M, Garamus VM, Dias FB, Almásy L, Galbrecht F, Charas A, et al. Macromolecules 2006;39:6505.

[11] Somma E, Loppinet B, Chi C, Fytas G, Wegner G. Phys Chem Chem Phys 2006; 8:2773.

[12] Dias FB, Knaapila M, Monkman AP, Burrows HD. Macromolecules 2006;39:1598.

[13] Wu L, Sato T, Tang H-Z, Fujiki M. Macromolecules 2004;37:6183.

[14] Banach MJ, Friend RH, Sirringhaus H. Macromolecules 2004;37:6079. 
[15] Grell M, Bradley DDC, Long X, Chamberlain T, Inbasekaran M, Woo EP, et al. Acta Polym 1998;49:439.

[16] Chen SH, Su AC, Su CH, Chen SA. Macromolecules 2005;38:379.

[17] Chen SH, Su AC, Chen SA. J Phys Chem B 2005;109:10067.

[18] Kitts CC, Vanden Bout DA. Polymer 2007;48:2322.

[19] Rahman MH, Chen C-Y, Liao S-C, Chen H-L, Tsao C-S, Chen J-H, et al. Macromolecules 2007;40:6572

[20] Knaapila M, Dias FB, Garamus VM, Almásy L, Torkkeli M, Leppänen K, et al. Macromolecules 2007;40:9398.

[21] Schmidt MW, Baldridge KK, Boatz JA, Elbert ST, Gordon MS, Jensen JH, et al. J Comput Chem 1993:14:1347.

[22] Becke AD. J Chem Phys 1993;98:5648.

[23] Lee C, Yang W, Parr RG. Phys Rev B 1988;37:785.

[24] Hariharan PC, Pople JA. Theor Chim Acta 1973;28:213.
[25] Malkin VG, Malkina OL, Salahub DR. Chem Phys Lett 1993:204:80.

[26] Malkin VG, Malkina OL, Salahub DR. Chem Phys Lett 1993;204:87.

[27] St-Amant A, Salahub DR. Chem Phys Lett 1990;169:387.

[28] Perdew JP, Chevary JA, Vosko SH, Jackson KA, Pederson MR, Singh DJ, et al. Phys Rev B 1992;46:6671.

[29] Perdew JP, Wang Y. Phys Rev B 1992;45:13244.

[30] Pedersen JS, Schurtenberger P. Macromolecules 1996;29:7602.

[31] Ou-Yang W-C, Chang C-S, Chen H-L, Tsao C-S, Peng K-Y, Chen S-A, et al. Phys Rev E 2005;72:031802.

[32] Li Y-C, Chen K-B, Chen H-L, Hsu C-S, Tsao C-S, Chen J-H, et al. Langmuir 2006; 22:11009.

[33] Burrows HD, Knaapila M, Monkman AP, Tapia MJ, Fonseca SM, Ramos ML, et al J Phys Condens Matter 2008;20:104210.

[34] Ranger M, Rondeau D, Leclerc M. Macromolecules 1997;30:7686. 